\title{
Giant intra-abdominal cystic lymphangioma
}

\author{
Fatih Altintoprak ${ }^{1}$, Mustafa Yener Uzunoglu², Kayhan Ozdemir ${ }^{2}$, Enis Dikicier ${ }^{2}$, and Zeynep Kahyaoglu
}

${ }^{1}$ Department of General Surgery, Sakarya University Faculty of Medicine, Sakarya; Departments of ${ }^{2}$ General Surgery and ${ }^{3}$ Pathology, Sakarya University Research and Educational Hospital, Sakarya, Turkey

Received: January 21, 2017

Revised : February 23, 2017

Accepted: July 15, 2017

Correspondence to

Fatih Altintoprak, M.D.

Tel: +90-533-548-3415

Fax: +90-264-275-9192

E-mail: fatihaltintoprak@yahoo.com
A 53-year-old male patient was evaluated with 2-year-old abdominal pain, postprandial vomiting and abdominal distension. In physical examination, an intra-abdominal mass which is full-filling of the left upper and lower quadrant of abdomen with unclear border was palpated. On abdominal magnetic resonance imaging, it was determined that the giant intra-abdominal lesion which has cystic and homogeneous in-nature, multiloculated and including multiseptations with $303 \times 226 \times 235 \mathrm{~mm}$ in diameter extende ing from the left diaphragm to the pelvis (Fig. 1A). Intra-abdominal giant-cystic lymphangioma (CL) was considered as preliminary diagnosis. In operation, cyst was excised complete with open surgery because it was unsuitable for laparoscopic surgery by the reason of lesion size (Fig. 1B). In histopathologic examination; dilated lymphatic vessels filled with lymphatic material and flattened endothelial cells without atypia which are typical for CL were detected (Fig. 2).

The CL is a rare benign tumor of the lymphatic system. The most accepted theory for the development of CL is a congenital anomaly. According to this theory, CLs are the result of incorrect connections between lymphatic channels and venous system during embryogenesis. CLs are usually asymptomatic but may be symptomatic depending on their location and size. Differential diagnosis from some cystic lesions is very important, such as hydatid disease (may require medical
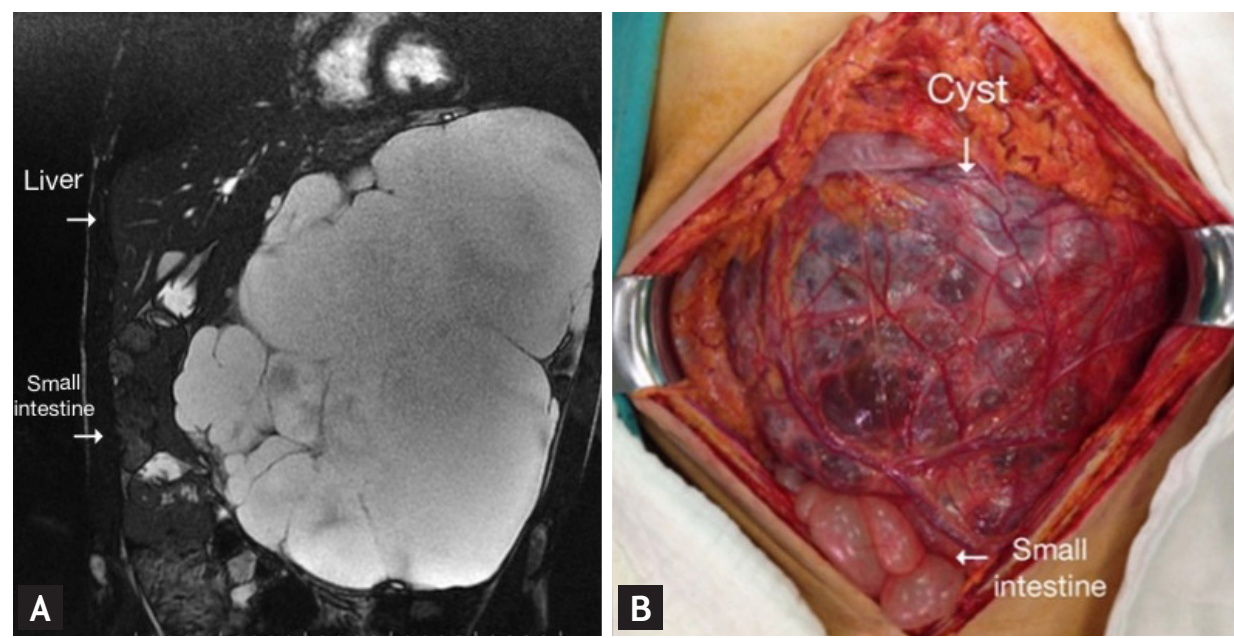

Figure 1. Abdominal magnetic resonance image (MRI) and intraoperative view. (A) Abdominal MRI shows giant intra-abdominal lesion which has cystic and homogeneous innature, multiloculated and including multiseptations. (B) Intraoperative views shows an intra-abdominal cystic mass. 

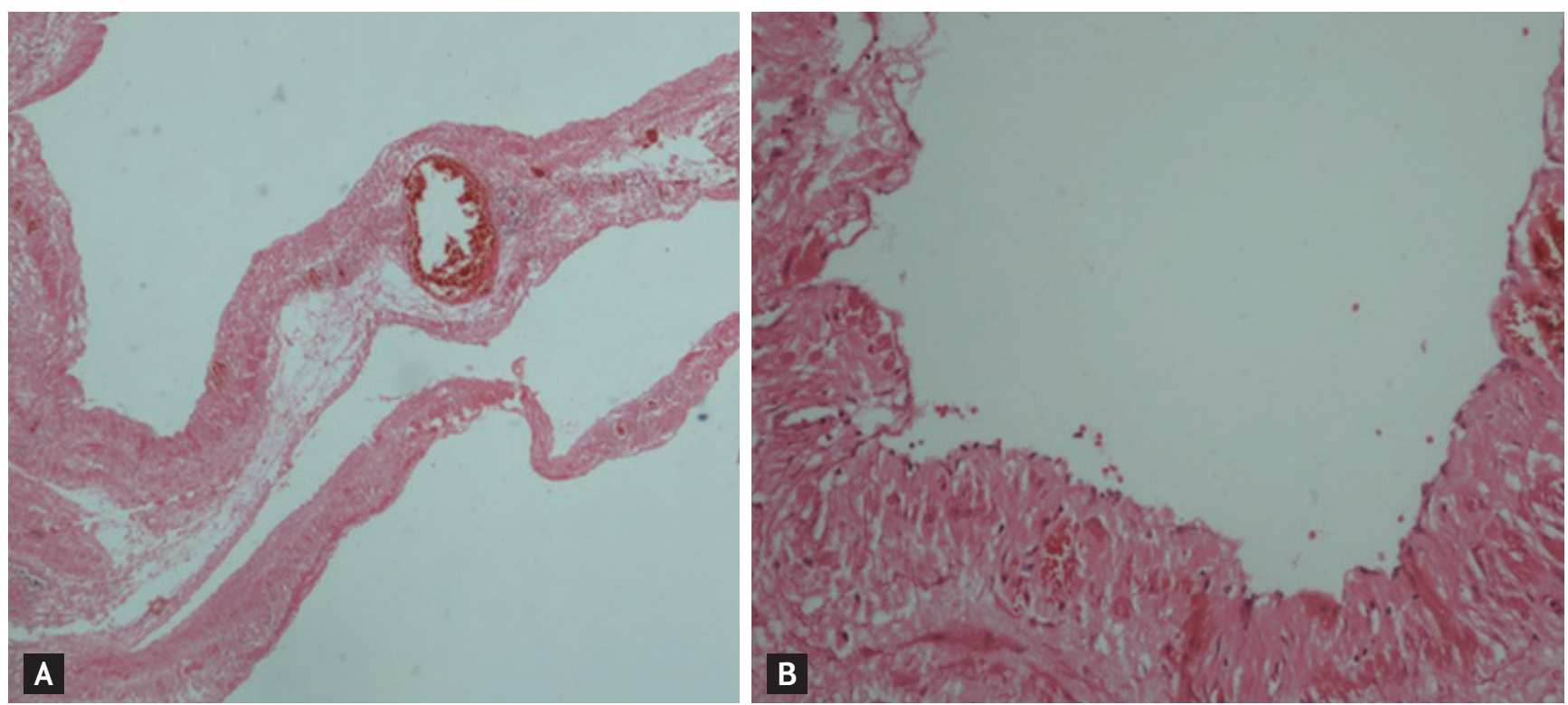

Figure 2. Microscopic findings. Dilated lymphatic vessels filled with lymphatic material and flattened endothelial cells without atypia (A: H\&E, ×10; B: H\&E, ×40).

treatment) or mesenteric cysts (malignant potential). For diagnosis and differential diagnosis, magnetic resonance imaging is better than the other imaging tools for clarifies the nature of the contents of the cysts. Percutaneous interventions (aspiration with or without injection of sclerosing agents) have high recurrence rate in intra-abdominal CLs. Complete surgical excision of cyst is recommended treatment in these lesions if there is no contraindication for surgical intervention. Laparoscopic surgery may be prefer in selected patients.

Written informed consents were obtained.

\section{Conflict of interest}

No potential conflict of interest relevant to this article was reported. 